\title{
An Evaluation of Non-Surgical Periodontal Therapy in Patients with Rheumatoid Arthritis
}

\author{
Caio V.G. Roman-Torres ${ }^{1,2, *}$, José S. Neto ${ }^{2}$, Marcio A. Souza ${ }^{2}$, Humberto O. Schwartz-Filho ${ }^{1}$, \\ William C. Brandt ${ }^{1}$ and Ricardo E.A.S. Diniz ${ }^{3}$
}

\author{
${ }^{I}$ Department of Dentistry, University of Santo Amaro, SP, Brazil; ${ }^{2}$ Department of Periodontology, Metropolitan Univer- \\ sity of Santos, SP, Brazil, ${ }^{3}$ Department of Medicine, Metropolitan University of Santos, SP, Brazil
}

\begin{abstract}
The aim of this study was to evaluate the efficacy of periodontal scaling and oral hygiene instruction for patients with mild chronic periodontitis and rheumatoid arthritis through clinical periodontal parameters and laboratory tests for CRP (C- reactive protein) and ESR (erythrocyte sedimentation rate). Twelve individuals with rheumatoid arthritis and 12 healthy individuals were evaluated, with a mean age of 45.38 and 46.75 respectively, all female and with mild, chronic periodontitis. The participants were evaluated clinically and periapical radiographs were taken (T1), after which periodontal treatment was instituted. After ninety days (T2), new clinical and laboratory data were obtained. Probing depth, bleeding index, and plaque indexes were observed in both groups, and the results demonstrated reductions but no statistical differences. Laboratory tests for CRP and ESR produced higher values for the rheumatoid arthritis group with T1- T2 reductions on the average, but the values were still higher than in the health group. We conclude that periodontal therapy in patients with rheumatoid arthritis and mild chronic periodontitis showed a improvement in the periodontal clinical parameters and laboratory tests that were evaluated.
\end{abstract}

Keywords: Dental scaling, periodontal debridement, periodontitis, rheumatoid arthritis.

\section{INTRODUCTION}

Rheumatoid arthritis (RA) is a systemic, inflammatory disease that is chronic and progressive, which affects primarily the synovial membrane of the joints and which may lead to the destruction of bone and cartilage. Records indicate that the occurrence rate of this disease in the adult population is about $1 \%$ in all ethnic groups, predominantly in females where it is two to three times more prevalent compared to males [1]. A multi-centered, Brazilian population sample of macro-regions of the country found a prevalence of RA of up to $1 \%$ in the adult population, which corresponds to an estimated 1.3 million people affected. The diagnosis of RA is established by considering clinical findings and laboratory tests. No single test, whether laboratory, imaging, or histopathology, confirms the diagnosis [2].

Chronic inflammatory disease and periodontal disease (PD) are both characterized by the dysregulation of the carrier inflammatory response by means of increasing the secretion of inflammatory mediators thus causing the destruction of the periodontium and the synovium [3, 4]. Some clinical and epidemiological studies have shown a high prevalence of periodontitis and tooth loss in patients with RA. We suggest that periodontitis is a possible triggering factor and maintainer of autoimmune inflammatory response in RA patients [5].

*Address correspondence to this author at the Rua Waldomiro Silveira 18, apto 71, CEP: 11065-200 Santos/SP - Brazil; Tel: +55 1399130 4747;

E-mail:cvgrt@hotmail.com
The theory that explains the association between RA and PD involves the anti-cyclic, citrullinated peptide (anti -CCP) and the citrullinated peptide (CCP), which are generated by a post- transcriptional modification (citrullination) of the peptidylarginine deiminase. Porphyromonas gingivalis is a one of the major microorganisms involved in PD and the only known bacterium that releases the enzyme, and therefore, it has been associated with RA [6].

To evaluate the periodontal status in patients with RA, Ishi et al. [7] examined 39 patients with RA (test group) and 22 healthy patients. They observed that patients with RA had fewer teeth, a higher prevalence of sites with dental plaque, and a higher frequency of sites with periodontal attachment loss. Based on these results, they suggested that there is an association between periodontal disease and RA. In 2010, Dissick et al. [8] conducted a study on 91 patients with RA and 41 patients with osteoarthritis who served as controls. Clinical examinations were performed and panoramic radiographs were examined to assess the presence and severity of periodontics. They concluded that PD is the most common and most severe in patients with RA and that further studies are needed for patients to understand the relationship between the two conditions.

In 2013, Erciyas et al. [9] evaluated individuals with RA and PD. Patients were monitored at baseline and at three months after periodontal therapy. Serum values for ESR (erythrocyte sedimentation rate) and CRP (C- reactive protein) were evaluated before and after non-surgical periodontal therapy. At the end of 3 months, they showed a reduction 
in the values of the tests. They concluded that periodontal therapy may be beneficial in reducing the severity of RA serum measures derived from ESR and CRP tests in patients with RA and PD.

Given the remarks cited, being aware of the significance of the need to understand the relationship between periodontal disease and systemic diseases, this research is aimed to evaluate the prevalence of the disease as well as possible clinical and laboratory relations between the two pathologies.

\section{MATERIALS AND METHODS}

This longitudinal study was approved by the Research Committee of the Metropolitan University of Santos, and all individuals had access to the terms of consent. A total of 56 individuals with rheumatoid arthritis were assessed, and we selected 12 women with a mean age of 45.38 who had been diagnosed with RA 10 years earlier and who had also slight chronic periodontitis [10]. All of the patients were referred from the outpatient specialty medicals service and the rheumatologist's specialty outpatient clinic of the Metropolitan University of Santos diagnosed and evaluated individuals in the test group and the control group. The control group was composed of individuals who initiated periodontal treatment, did not have any systemic involvement, and had probing depth compatible with the individuals in the test group. The criteria for participation in the study included not having any dental procedure performed in the last six months, not having used antibiotic medication in the last six months, and no smokers.

The main parameters for the diagnosis of RA include counting the number of painful joints and the number of swollen joints, inflammatory activity (ESR and CRP), assessment of pain intensity, and assessment of joint mobility and functional capacity [11]. Before the start of periodontal treatment, these tests were performed, and blood tests (ESR and CRP) were requested and evaluated by the rheumatologist in charge. The healthy subjects (control group) also underwent blood tests.

Upon completion medical history evaluation, periodontal clinical examinations were performed by a previously trained and calibrated periodontist, and in the sample studied, $10 \%$ were examined twice for each of the clinical criteria in order to obtain the intra-examiner reliability as measured by Kappa statistics (0.87).

We observed the clinical parameters of periodontal probing depth (PPD) in six points per tooth, and plaque index (PI) and bleeding on probing index (BOP) [12] conducted dichotomously for buccal, palatal/lingual, midbuccal, distobuccal, midpalatal or lingual and disto palatal or lingual All of the participants received radiographic, periapical, dental examinations in order to confirm the clinical exam and to make the diagnosis.

Subjects received oral hygiene instructions and underwent therapy for full mouth disinfection, to prevent reinfection of the untreated sites, and they followed the protocol of holding two sessions with an interval of less than 24 hours between sessions. At the beginning of each session, oral hygiene instructions were given to each subject indi- vidually and we tried to teach patients the correct way of using a brush and floss. The treatment performed included full mouth debridement by a single periodontist who was blinded to the participants and the data obtained in clinical trials [13].

Each participant received seven doses of the individuallybased mouthwash chlorhexidine $0.12 \%$, to be used within seven calendar days after execution of the first session of the proposed therapy, and they were told to always use it after brushing the last thing at night and then to wait 30 minutes to receive the maximum effectiveness of the chlorhexidine, because the surfactant in the toothpaste interferes with the active ingredients in the mouth wash. Three months after the last treatment clinical examination and laboratory (ESR and CRP) tests were performed. Then the participants were referred to clinics operating in the college of Dentistry UNIMES as needed.

After tabulating the clinical and laboratory data for the participants included in this study, the data were subjected to statistical analysis. For this, the software SPSS 13.0 and Bioestat 5.0 were used. In all analytical situations, a significance level of $95 \%$ should be adopted. For each cluster of analytical interest, the characteristic distribution of the sample was tested and among the selected statistical tests being used were the statistical tests ANOVA with two factors (factor "health condition" - healthy or rheumatoid factor and "periodontal treatment" - Immediate evaluation or after periodontal treatment) and the Tukey test.

\section{RESULTS}

The results indicated that there was no significant interaction between the factors, "health condition" and "periodontal treatment" regardless of the parameter evaluated (Table 1).

When we evaluated only the factor, "periodontal treatment," the periodontal parameters PPD and BOP and the blood parameters ESR and CRP did not achieve statistical differences independent of periodontal treatment or they may not have been realized. However, PI showed higher parameter values before periodontal treatment.

When we evaluated only the factor, "health condition" of the patient, only the parameter PPD showed no difference in outcomes between healthy patients and those with rheumatoid arthritis. In the evaluation of all other parameters (ESR, CRP, PI, and BOP), patients with rheumatoid arthritis had higher values of these parameters than healthy patients.

\section{DISCUSSION}

An association between PD and RA has been considered since 1820, when the treatment of extraction was the most commonly executed. Epidemiological studies suggest that the prevalence of RA and PD is similar and reaches $5 \%$ of the population aged over 50 years. Both are chronic diseases that may influence environmental, immunologic, and genetic factors leading to tissue and bone destruction during periods of outbreaks of periodontal disease [14]. Individuals with RA can have greater difficulties with oral hygiene due to temporomandibular joint pain and limitations in the movements of the hands [15]. 
Table 1. Mean and standard deviation of periodontal parameters and laboratory tests from healthy individuals (control group) and rheumatoid arthritis (test group) before (T1) and after periodontal treatment (T2).

\begin{tabular}{|c|c|c|c|}
\hline PPD (mm) & Control & $2,1(0,3)$ & $1,9(0,2)$ \\
\hline \multirow{2}{*}{ PI (\%) } & Control & $26,4(7,1)$ & $21,0(2,7)$ \\
\hline & Test & $34,4(6,6)$ & $29,4(4,8)$ \\
\hline BOP (\%) & Test & $23,4(5,0)$ & $20,2(5,4)$ \\
\hline \multirow[b]{2}{*}{ CRP } & Control & $0,2(0,1)$ & $0,1(0,0)$ \\
\hline & Test & $4,1(5,6)$ & $3,2(4,4)$ \\
\hline
\end{tabular}

PPD - periodontal probing depth, PI - plaque index, GI - gingival index, CRP - C-reactive protein, ESR - erythrocyte sedimentation rate, BOP - bleeding on probing

A large number of epidemiological, case-control and cohort research projects have studied the relationship between PD and RA [16-19]. A higher incidence of missing teeth, biofilm accumulation, and greater probing depths are often observed in patients with RA. There are problems in comparing studies due to a lack of standardization in the concept of periodontal disease in the characterization and time of individuals with RA, the medications used daily in patients with RA, and the inclusion of smokers in the groups tested. In some studies, there was a higher prevalence of severe periodontitis in patients with RA [8,20], and in some studies, this was not observed [4] but in both cases the number of individuals with missing teeth or edentulous total was always higher in patients with RA.

In our study, the participants included had clinical conditions that were very similar in both groups (test and control) so they could be compared. The inclusion of test and control groups with a probing depth average of $2.3 / 2.1 \mathrm{~mm}$, respectively, does not allow a conclusion on the effectiveness of periodontal treatment, as there was clinical improvement, but without statistical significance due to the low values of PPD. Results may be different if patients with moderate to severe disease were compared, i.e. average probing depths $\geq 5 \mathrm{~mm}$ or CAL $\geq 4 \mathrm{~mm}$. The improvement in the parameters PI and BOP was mainly the result of the oral hygiene instructions that were constantly repeated at all visits. On the other hand, even with guidelines, some patients in advanced RA lose some hand movement preventing proper brushing or even preventing flossing $[8,21]$, which must be mitigated with the use of brushes and floss mounted in a plastic frame.

The diagnosis of RA is made by means of a combination of clinical, laboratory, and radiographic findings, and individuals included in the study had a confirmed diagnosis of RA. ESR and CRP tests that are called acute phase reactants were used to establish "baselines" and subsequently monitor the evolution of infectious diseases including autoimmune diseases and others $[22,23]$. The participants in our study had a diagnosis of RA and their predetermined serum levels of ESR and CRP were used as markers for monitoring systemic inflammation, as were elections for control of the inflammatory response. Although slight, there was a decrease in both serum levels evaluated in the test group, which was similar to the study [9].

There was no statistical difference, probably due to the fact that the individuals in the test group presented results for these tests that were widely discrepant. When assessed in the control group, these tests showed normal results after performing periodontal therapy. A statistical difference was observed when comparing the individuals in the test group and the control group indicating that individuals with RA had higher values of ESR and CRP.

Non-surgical periodontal therapy can promote an improvement in clinical periodontal parameters, contributing to a slight improvement of the clinical symptoms of RA [1]. Therapeutic procedures were performed in two sessions in 24 hours, with good acceptance by patients who reported no discomfort or pain symptoms. All subjects received oral hygiene instructions at all visits, which was essential for the control of biofilm influencing the plaque index and bleeding. We believe that oral hygiene instruction and demonstration sessions on how and where to floss properly are fundamental. Professionals and their patients should no longer face problems like periodontal disease restricted to the oral cavity, as there is a possibility that it has a negative impact on general health: a healthy mouth contributes to a healthy body.

\section{CONCLUSION}

We conclude that periodontal therapy in patients with rheumatoid arthritis and mild, chronic periodontitis patients showed improvement in clinical efficacy after having been evaluated with clinical periodontal parameters and laboratory tests. However, the differences in inflammatory markers were not statistically significant within either group from baseline to three months. 


\section{CONFLICT OF INTEREST}

The authors confirm that this article content has no conflict of interest.

\section{ACKNOWLEDGEMENTS}

Declared none.

\section{REFERENCES}

[1] Monsarrat P, Vergnes JN, Cantagrel A, et al. Effect of periodontal treatment on the clinical parameters of patients with rheumatoid arthritis: study protocol of the randomized, controlled ESPERA trial. Trials 2013; 14: 253 .

[2] Mota LM, Cruz BA, Brenol CV, et al. Guidelines for the diagnosis of rheumatoid arthritis. Rev Bras Reumatol 2013; 53(2): 141-57.

[3] Bingham CO III, Moni M. Disclosures. periodontal disease and rheumatoid arthritis. the evidence accumulates for complex pathobiologic interactions. Curr Opin Rheumatol 2013; 25(3): 345-53.

[4] Reichert S, Haffner M, Keyber G, et al. Detection of oral bacterial DNA in synovial fluid. J Clin Periodontol 2013; 40(6): 591-8.

[5] Goeldner I, Skare Tl, Reason ITM, Utiyama SRR. Artrite reumatoide: uma visão atual. Bras Patol Med Lab 2011; 47(1): 495-503.

[6] Liao F, Li Z, Wang Y, Shi B, Gong Z, Cheng X. Porphyromonas gingivalis may play an important role in the pathogenesis of periodontitis-associated rheumatoid arthritis. Med Hypotheses 2009; 72(6): $732-5$.

[7] Ishi EP, Bertolo MB, Rossa C Jr, Kirkwood Kl, Onofre MA. Periodontal condition in patients with rheumatoid arthritis. Braz Oral Res 2008; 22(1): 72-7.

[8] Dissik A, Redman RS, Jones M, et al. Association of periodontitis with rheumatoid arthritis: a pilot study. J Periodontol 2010; 81(2): 223-30.

[9] Erciyas K, Sezer U, Ustün K, et al. Effects of periodontal therapy on disease activity and systemic inflammation in rheumatoid arthritis patients. Oral Dis 2013; 19(4): 394-400.
[10] Armitage GC. Development of a classification system for periodontal diseases and conditions. Ann Periodontol 1999; 4(1): 1-6.

[11] Block SR. Hidden hazards and practical problems: comment on the 2002 update of the American College of Rheumatology Guidelines for the Management of Rheumatoid Arthritis. Arthritis Rheum 2002; 46(11): 3102-3.

[12] Ainamo J, Bay I. Problems and proposals for recording gingivitis and plaque. Int Dent J 1975; 25(4): 229-35.

[13] Chan YK, Needleman IG, Clifford LR. Comparison of four methods of assessing root surface debridement. J Periodontol 2000; 71(3): 385-93.

[14] El-Shinnawi U, Soory M. Associations between periodontitis and systemic inflammatory diseases: response to treatment. Recent Pat Endocr Metab Immune Drug Discov 2013; 7(3): 169-88.

[15] Persson GR. Rheumatoid arthritis and periodontitis - inflammatory and infectious connections. Review of the literature. J Oral Microbiol 2012; 4 .

[16] Mercado F, Marshall RI, Klestov AC, Bartold PM. Is there a relationship between rheumatoid arthritis and periodontal disease? J Clin Periodontol 2000; 27(4): 267-72.

[17] Mayer Y, Balbir-Gurman A, Machtei EE. Anti-tumor necrosis factor-alpha therapy and periodontal parameters in patients with rheumatoid arthritis. J Periodontol 2009; 80(9): 1414-20.

[18] Berthelot JM, Le Goff B. Rheumatoid arthritis and periodontal disease. Joint Bone Spine 2010; 77(6): 537-41.

[19] Han JY, Reynolds MA. Effect of antirheumatic agents on periodontal parametersand biomarkers of inflammation: a systematic review and meta-analysis. J Periodontal Implant Sci 2012; 42(1): 3-12.

[20] Joseph R, Rajappan S, Nath SG, Paul BJ. Association between chronic periodontitis and rheumatoid arthritis: a hospital-based case-control study. Rheumatol Int 2013; 33(1): 103-9.

[21] Torkzaban P, Hjiabadi T, Basiri Z, Poorolajal J. Effect of rheumatoid arthritis on periodontitis: a historical cohort study. J Periodontal Implant Sci 2012; 42(3): 67-72.

[22] Aletha D, Neogi T, Silman AJ, et al. Rheumatoid arthritis classification criteria. Arthritis Rheum 2010; 62 (9): 2569-81.

[23] Aguiar FJB, Ferreira-Júnior M, Sales MM, et al. Proteína C reativa: aplicações clínicas e propostas para utilização racional. Rev Assoc Med Bras 2013; 59(1): 85-92.

\author{
Received: March 09, 2015 \\ Revised: April 01, 2015 \\ Accepted: April 13,2015 \\ (C) Roman-Torres et al.; Licensee Bentham Open.
}

This is an open access article licensed under the terms of the Creative Commons Attribution Non-Commercial License (http://creativecommons.org/licenses/by-nc/3.0/) which permits unrestricted, non-commercial use, distribution and reproduction in any medium, provided the work is properly cited. 